\title{
Retinal Vessel Segmentation Using an Entropy-Based Optimization Algorithm
}

\author{
Sukhpreet Kaur, IKGPTU, Kapurthala, India \\ Kulwinder Singh Mann, Guru Nanak Dev Engineering College, Ludhiana, India
}

\begin{abstract}
This article presents an algorithm for the segmentation of retinal blood vessels for the detection of diabetic retinopathy eye diseases. This disease occurs in patients with untreated diabetes for a long time. Since this disease is related to the retina, it can eventually lead to vision impairment. The proposed algorithm is a supervised learning method of blood vessels segmentation in which the classification system is trained with the features that are extracted from the images. The proposed system is implemented on the images of DRIVE, STARE and CHASE_DB1 databases. The segmentation is done by forming clusters with the features of patterns. The features were extracted using independent component analysis and the classification is performed by support vector machines (SVM). The results of the parameters are grouped by accuracy, sensitivity, specificity, positive predictive value, false positive rate and are compared with particle swarm optimization (PSO), the firefly optimization algorithm (FA) and the lion optimization algorithm (LOA).
\end{abstract}

\section{KEYWORDS}

Diabetic Retinopathy, Feature Extraction, Optimization, Retinal Vessels

\section{INTRODUCTION}

The structure of blood vessels in retina helps in detection of number of eye diseases which includes arteriosclerosis, diabetes, retinal vein occlusion, retinal artery occlusion, hypertension, cataract, glaucoma and most importantly diabetic retinopathy. These all diseases can be detected by monitoring the changes in the structure of an eye. A human eye consists of iris, lens, blood vessels, pupil, retina etc. Eye helps in sensing and visualizing different objects. All the different parts of an eye help in visualizing in one or another way. Each and every part can lead to different disease if affected by diabetes. The patients having prolonged and untreated diabetes suffered from eye disease named as diabetic retinopathy (DR). DR is the leading cause of blindness as the retina of the eye is directly affected by this disease. According to the latest figures issued by World Health Organization (WHO), the patients suffering from diabetes will reach to 300 million by 2025 (Zimmet, 2016). Currently, the number of diabetics are 69.2 million from which 7 million people suffer from vision loss (Joshi, 2016).

Diabetes can affect any body part like eyes, kidneys, the liver, the heart, and bones. Eye became the significant part of the human organ system needs special care. The impact of untreated blindness

\section{DOI: 10.4018/IJHISI.2020040105}

This article, originally published under IGI Global's copyright on December 20, 2019 will proceed with publication as an Open Access article starting on January 14, 2021 in the gold Open Access journal, International Journal of Healthcare Information Systems and Informatics (converted to gold Open Access January 1, 2021), and will be distributed under the terms of the Creative Commons Attribution License (http://creativecommons.org/licenses/by/4.0/) which permits unrestricted use, distribution, and production in any medium, provided the author of the original work and original publication source are properly credited. 
is shown on blood vessels, nerves and the vision of the patient. DR is caused when the blood vessels of the human retina are damaged, and they leaked blood and lipids. The symptoms of DR is same as that of changes that occur in eyes due to age, so there is a need of fine procedures which can differentiate between DR and age related eye degradation.

DR can be detected easily by segmenting retina and its blood vessels. The segmented images of the retina help in studying the blood circulation of human eye at the micro level. As it is the part of central nervous system, so it is easy for researchers as well as for the ophthalmologists to study the retina for different pathologies (Fraz, 2012). It is highly sensitive to light and consists of optic disc, blood vessels and macula. The various pathologies in the retina of an eye can be detected by monitoring the variations in the various components of retina. The blood vessels can be easily visible to the human eye. So, the pathologies in blood vessels can be checked easily by the clinicians. All the different eye diseases that occur due to pathologies in retina can be detected by segmentation of retina. The retinal images are captured using special camera named as Fundus Camera as well as by using ophthalmoscopes. Fundus camera is a camera of high resolution especially used for retinal imaging. Other techniques used for acquiring retinal images include laser screening, optics screening and angiography. Fundus imaging is prominently used for retinal imaging by dilating the pupil of retina using some eye drops. Then the fundus of the image which is the region opposite to lens of eye and includes optic disc and macula is focused for imaging.

The various diseases in eye cause different types of changes in the vasculature of human retina. The various disorders of an eye can be checked by studying the segmentation of retina and its various parts. The clinicians also study the changes in the retinal vasculature for evaluating the severity of eye diseases and to decide whether the disease can be curable or not. The various changes in the retinal eye can be categorized into neovascularization, collateralization and origination of retinal vascular shunt (Paul, 1974). If the new blood vessels are originated in either retina or in the area adjacent to it, then it leads to neovascularization. In this the blood vessels grows in irregular fashion generally near larger arteries and veins in any direction. They are appeared in the areas where there are no blood vessels present. Collateralization relates to the growth of blood vessels in between the existing blood vessels by joining new arteries and veins to the existing new arteries and veins respectively. The blood flow in the vessels is hampered if there is cross connection between them. The last case of formation of shunt occurred when the blood flows without using capillary bed at a very high speed.

DR is disorder of human eye which is caused by untreated diabetes. In this the blood vessels are damaged and they leak blood and in some cases they lead to growth of new blood vessels. Due to which, the vision deteriorates and it leads to blindness. DR leads to formation of microaneurysms, exudates, hemorrhages, cotton wool spots, and lesions. Microaneurysms are small red dots which are formed when the walls of capillary blood cells are weakened. When the weakened blood cells leak, they become hemorrhages which are flame-shaped. After that, when the proteins and lipids from the blood are leaked, then they lead to formation of exudates. Hard exudates are of yellow or white color in eye retina. When the severity of DR advances, the blood vessels get obstructed and leads to soft exudates or cotton wool spots and they are white in color.

DR is classified broadly into two stages: Proliferative DR (PDR) and Non-Proliferative DR (NPDR). NPDR is the initial stage of DR in which the damage of retinal blood vessels has just started. The three stages of NPDR are mild, moderate and severe (You, 2011). The mild stage of NPDR is the initial stage of NPDR and requires no treatment but the progression of the disease needs to be monitored strongly by the clinicians. In mild NPDR only microaneurysms occur or in some other cases hemorrhages can also occur. In moderate NPDR, cotton wools spots start appearing due to blockage of blood vessels that nourish the retina. In the case of severe NPDR, the growth of new blood vessels starts in an irregular fashion in the eye retina. Finally, vision loss or blindness occurs due to formation of new blood vessels and due to weakness of existing blood vessels in the retina.

DR can be detected by the segmentation of blood vessels of the retina. The variations in width, length, branching angle, vascular pattern, and tortuosity of the blood vessels can be helpful in detecting 
various eye diseases. Manual segmentation of the human retina is a tedious task and requires expertise. So, various computer-aided techniques have come into existence which helped ophthalmologists to detect eye diseases to help their patients from vision impairment. Although there are number of techniques, but there is always a way of improving the existing methods.

\section{BACKGROUND}

The existing techniques of retinal blood vessels segmentation was categorized into seven major categories named: a) machine learning or pattern classification techniques; b) matched filtering; c) mathematical morphological techniques; d) vessel tracing; e) multiscale approaches; f) model based approaches; and g) hardware-based approaches. The large number of existing techniques is under the category of pattern classification techniques which categorizes the extracted features either into vessels or non-vessels. The pattern classification techniques are further divided into supervised and unsupervised techniques. In the case of unsupervised techniques, the classification of vessels and non-vessels is done based on extracted patterns.

\section{Supervised Methods of Pattern Classification}

In case of supervised techniques, the training data or the ground truth images are available for training the system. The ground truth images are generally segmented by the experts or clinicians. The classification of blood vessels is done by comparing the features of extracted patterns and the patterns of ground truth images. Li, Zhenshen, Chao et al. (2017) proposed a supervised technique for blood vessel segmentation based on features given by length, width and intensity of the input images. Since these are the local descriptors of the image, so they helped in maintaining the edge information locally. Mustafa, Haniza and Wahida (2017) proposed a technique for the detection of diabetic retinopathy and glaucoma based on 7-dimensional feature vector that was hybrid of gray levels and moment invariants features. The classification of vessels and non-vessels was finally done by using decision trees. Shah, Tong, Ibrahima et al. (2017) proposed a technique that can find the abnormalities of human retina using regional and Hessian matrix descriptors. The total number of 24 features was extracted for the pattern recognition process and the classification was performed using linear minimum squared error method and this helped in achieving the accuracy of $93 \%$ approximately. GeethaRamani et al. (2016) proposed a supervised method for the segmentation of blood vessels based on both image processing and data mining. The supervised learning was performed by the combination of k-means clustering. The final segmented image was formed by using decision tree classification and image post-processing by mathematical morphology and connected component analysis. Rahim et al. proposed a technique for the detection of microaneuysms using features given by area, length, mean, perimeter, major and minor area length, standard deviation. The classification process was performed by using decision trees, k-nearest neighbors, support vector machine, RBF Kernal SVM. This method was highly helpful in detection of DR as microaneuysms exists during the initial stages of DR. Aslani and Sarnel (2016) compute the 17-dimensional feature vector for the segmentation of blood vessels. The classification was done using Random Forest (RF) classifier for dealing with both with homogeneous and heterogeneous data. RF classifier is based on decision trees and it was implemented in the proposed algorithm using 150 decision tree with a depth of 15 for each branch. Hatanaka, Samo, Tajima et al. (2016) proposed a supervised technique for the segmentation using autocorrelation method on shift invariant local features on neighbors of pixels. The classification process was done using neural networks of two types in which first neural network worked on 105 pixels while the second neural network worked on output of first neural network, filtering and transformation process. The advantage of this method is that it can segment the images with low contrast.

Saha, Naskar, and Chatterji (2016) proposed a technique for detection of DR using wavelet transform and neural network. The wavelet-based analyzer was used to analyze the segmented images with ground 
truth images. The feed forward neural network was used for classification of vessels and non-vessels in the segmented images. Rajput, Manza, Patwari et al. (2015) proposed a method to detect NPDR in which features were extracted using Haar and Symlet wavelets and they will extract the features for each symptom of DR. This process of feature extraction helps in classifying the input image into different stages of NPDR that is mild, moderate or severe by extracting patterns for each symptom separately. The classification was finally done by k-means clustering and statistical means. Tang, Lin, Yang et al. (2015) designed a feature vector of 94 dimensions for pattern recognition. The features include Gabor responses at different scales and dimensions. The classification process was performed using support vector machines (SVM). Franklin et al. gave a supervised technique using neural network for the segmentation of retinal blood vessels. The image was preprocessed using background normalization and the system was then trained using feed-forward perceptron neural network and the classification was done using back-propagation algorithm. Franklin and Rajan (2014) used artificial neural network for training. The extracted features include Gabor responses and moment invariant based features. The input image was preprocessed using different filtering techniques and the system was trained and classification was done. The image was also post processed after connecting different isolated points. Wang, Yin, Cao et al. (2014) proposed a technique using ensemble learning for the segmentation process. In this, two classifiers were used, random forest (RF) classifier and convolutional neural network (CNN) which act as a feature classifier and feature extractor, respectively. CNN works in two different sublayers as convolutional sublayer and subsampling sublayer for extraction of features. RF classifies the vessels using the majority voting process and using winner -takes-all decision strategy. Roychowdhury, Koozekanani and Parhi (2017) used a Gaussian Mixture Model (GMM) for the classification of prominent features. The features were extracted using gradients of first and second-order derivatives. The key feature of this proposed algorithm is that the input image is converted into binary image for extraction of features. Marin, Awuino, Gegundez et al. (2011) designed a 7-dimensional feature vector for the classification of input images. The extracted features include gray level and moment invariant based features. The input image was preprocessed using central light reflex removal, background homogenization methods. The training was done using neural networks by calculating probability map of each pixel. The classification was performed using thresholding process. Finally, the gaps were filled in post-processing phase using artifact filling process. Peng et al. proposed an algorithm that can segment both thin as well as wide vessels by using radial projection. The thin and narrow vessels were segmented using radial projections and the wide vessels were segmented using steering wavelet and semi supervised learning. Lupascu, Tegolo and Trucco (2010) used a feature-based Ada Boost classifier for the segmentation of retinal blood vessels. The constructed feature vector is of 41 dimensions including features of intensity, spatial and geometrical features. Xu and Luo (2010) proposed a segmentation process using wavelets and curvelets for both thin and thick vessels. The thin vessels were classified further using Hessian matrix and thick vessels by support vector machines. Osareh and Shadgar (2009) proposed a method for segmentation for colored images of retina using principal component analysis, Gaussian model and support vector machines. Anzalone, Bizzari, Parodi et al. (2008) proposed an algorithm consisting of two blocks in which first block was used for enhancement of images of blood vessels and second block was able to do image binarization, image cleaning after evaluating the optimized values of measures of performance (MOPs). Ricci et al. proposed a technique for segmentation of blood vessels using orthogonal line gradient detectors and support vector machines. Both supervised and unsupervised learning was performed for the detection of all blood vessels. Soares et al. used Gabor wavelet at different scales for feature extraction along with Bayesian classifier for classification of extracted vessels. Staal, Abràmoff, Niemeijer et al. (2004) proposed a method for segmentation of blood vessels from two-dimensional colored images. In this, the ridges and patches were extracted which was from the vessel centerlines which in turn helped in extraction of prominent features. The classification process was done by k-nn classifier. Sinthanayothin, Boyce, Cook et al. (1999) proposed a method for the detection of optic disc and blood vessels using multilayer neural network which consists of 200 input nodes and two output nodes. 


\section{PROPOSED ALGORITHM}

The proposed algorithm is carried out in number of steps given by:

1. Image Preprocessing

2. Image Segmentation

3. Feature Extraction

4. Feature Selection and Optimization

5. Objective and Clinical Evaluation

\section{Image Preprocessing}

All the existing algorithms for the segmentation of blood vessels suffered from the problems of non-uniform illumination since all the public databases available online need some type of preprocessing for enhancing the images. Other problems include the presence of various pathologies such as cotton wool spots, bright lesions, hard and soft exudates. The actual accuracy of segmentation depends upon the accurate classification of vessels and non-vessels which in turn depends upon the segmentation of all the blood vessels including both thick and thin vessels.

The input image was taken from the publicly available online databases named as DRIVE, STARE and CHASE databases. DRIVE (Digital Retinal Images for Vessel Extraction) consists of 40 images in which 20 images are in training set and 20 images are in test set. In the database, 13 images are of healthy retina and 7 images are of pathological retina. Figure 1a and $1 \mathrm{~b}$ show the image of normal and abnormal retina of DRIVE dataset. The ground truth images are also available which are segmented from three different observers manually. STARE (Structured Analysis of Retina) consists of 20 images in which 10 images are of healthy retina and 10 images of pathological retina. The ground truth images of two observers are also present. CHASE_DB1 (Child Heart and Health Study in England Set) consists of 28 fundus images of 14 school children by images both eyes of each child. The reference of the results is given by two observers by giving results of manual segmentation.

\section{Extraction of Green Channel}

The input image is RGB image, but only green color of the image has the higher contrast of blood vessels. The red channel of the image has the problem of over saturation and there is no effective

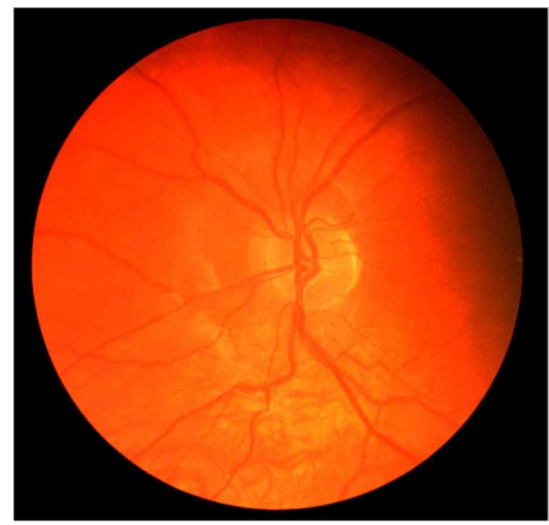


Figure 1b. Abnormal retina of DRIVE dataset

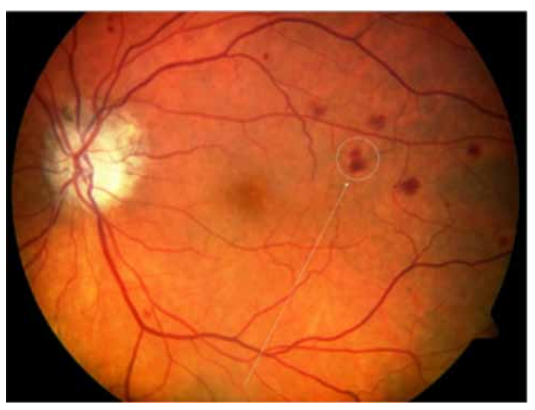

information in the blue channel. So, the first step in the preprocessing step is the extraction of green channel. Figure $2 \mathrm{a}, 2 \mathrm{~b}, 2 \mathrm{c}$ and $2 \mathrm{~d}$ shows the input image, corresponding red channel, green channel and blue channel.

\section{Conversion Into Gray Level}

The green channel of the RGB image is converted into gray level as gray level images requires less computation and less complex. Figure $3 \mathrm{a}$ and $3 \mathrm{~b}$ shows the green channel and corresponding gray scale image.

Figure 2a. Input image

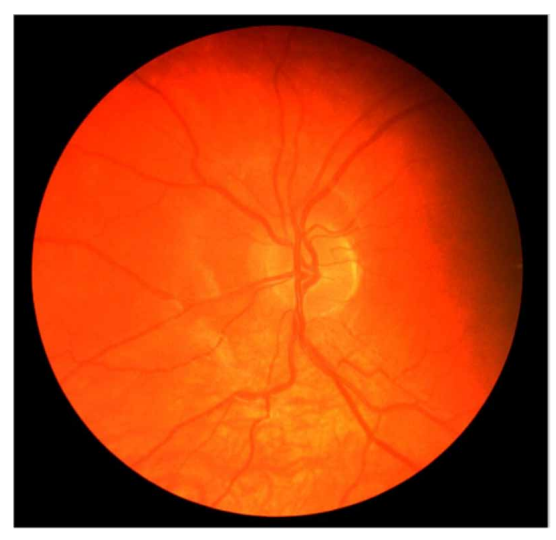

Figure 2b. Red channel

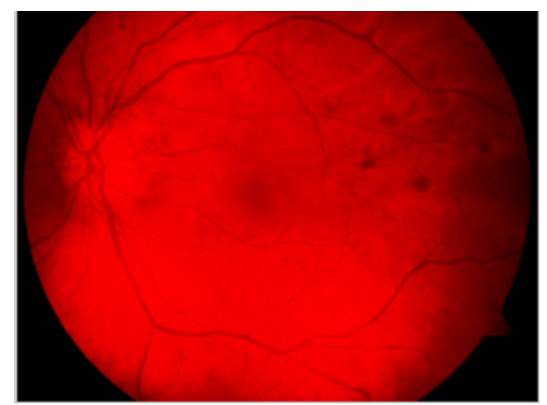


Figure 2c. Green channel

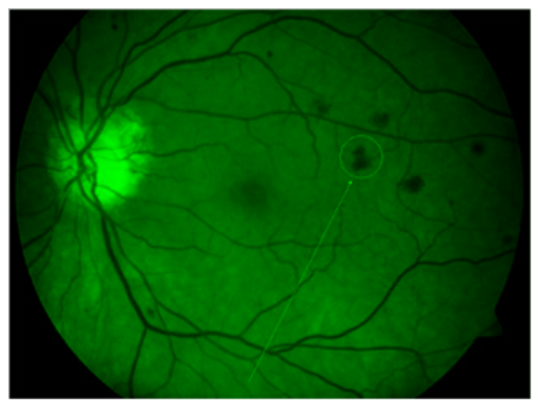

Figure 2d. Blue channel

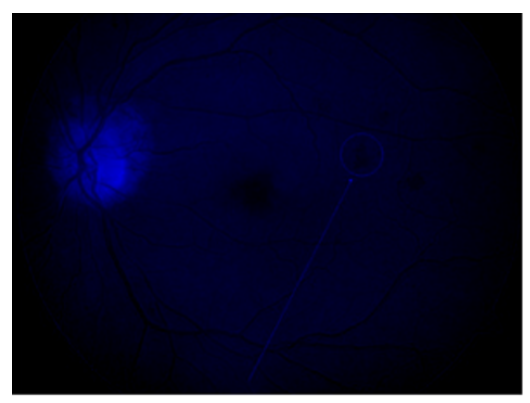

Figure 3a. Green channel

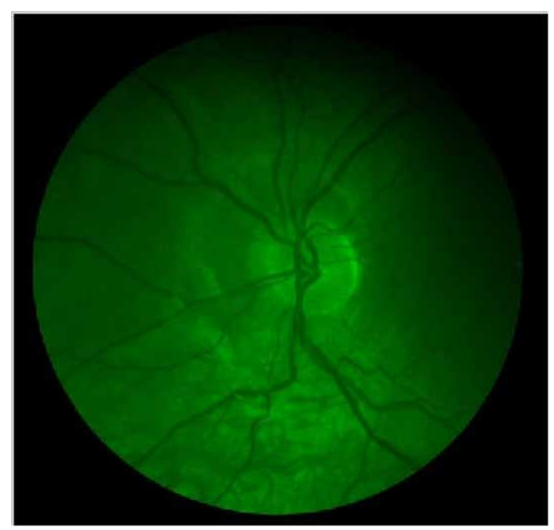

\section{Contrast Enhancement}

The appearance of the images can be increased by contrast of image by using Contrast Limited Adaptive Histogram Equalization (CLAHE) which enhances the image locally by dividing them into tiles. Figure $4 \mathrm{a}$ and $4 \mathrm{~b}$ show the input gray level image and corresponding CLAHE image.

\section{Image Filtering}

The image needs to be filtered using the gradient directional features. This filtering process will depict the edge strength. Figure $5 \mathrm{a}$ and $5 \mathrm{~b}$ show the corresponding input image and filtered image. 
International Journal of Healthcare Information Systems and Informatics

Volume 15 • Issue 2 • April-June 2020

Figure 3b. Gray level image

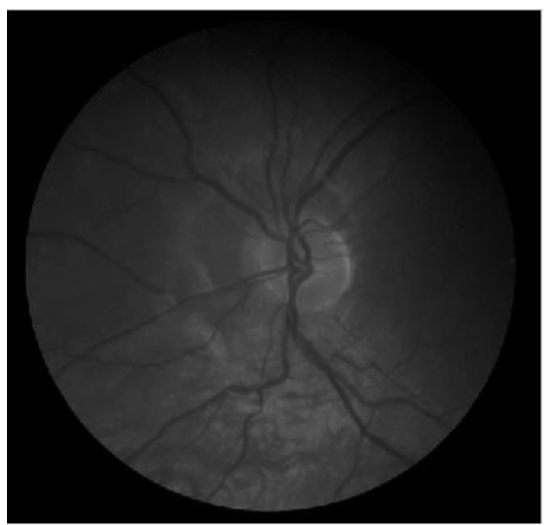

Figure 4a. Input image

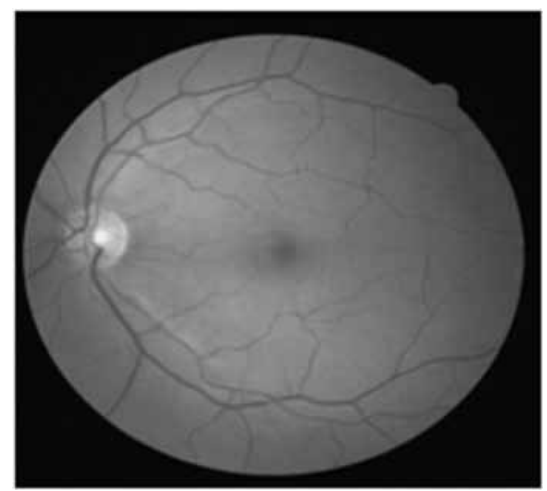

Figure 4b. CLAHE image

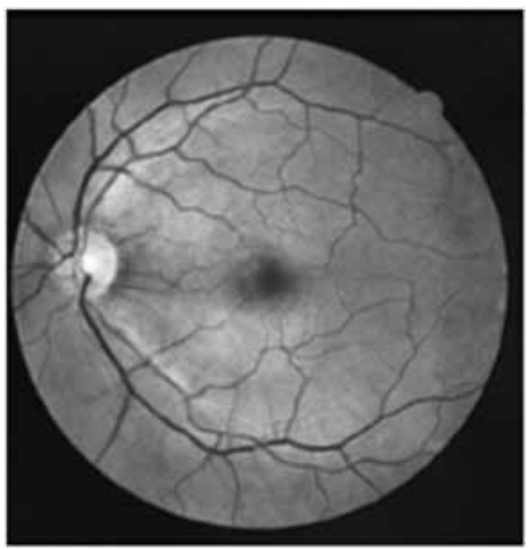


Figure 5a. Input image

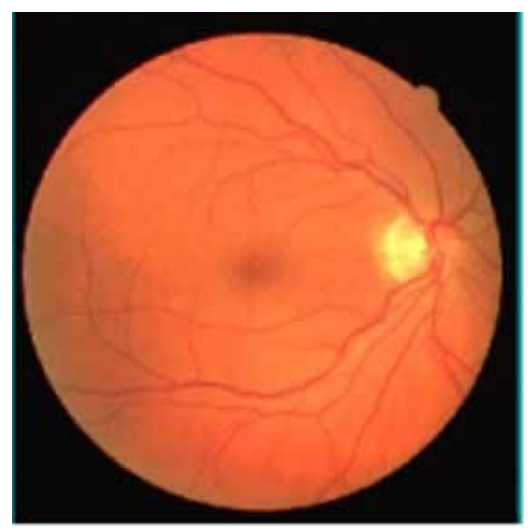

Figure 5b. Filtered image

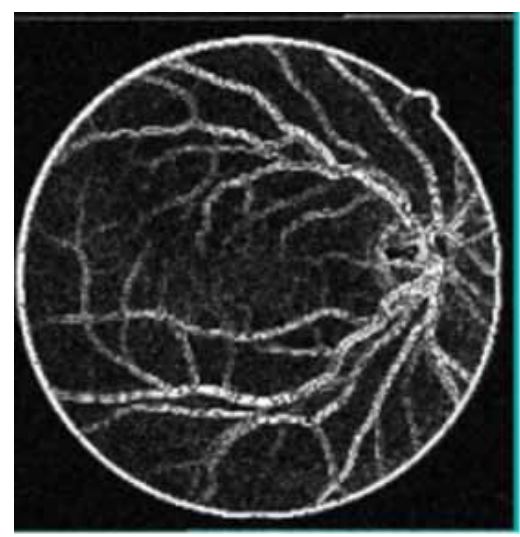

\section{Image Segmentation}

The segmentation of retinal blood vessels is done for analyzing the flow of blood in human eye retina. The segmentation process gives the best results if all the artifacts from them have been removed before the segmentation process. The preprocessing step of the proposed algorithm has extracted the green channel, enhanced the contrast and removed all the unwanted noise from the input image. There are number of methods by which segmentation can be performed which include the name of operators like Prewitt, Robert, Canny, different thresholding techniques, and edge-based detection techniques.

In the proposed algorithm, thresholding-based clusters were used for the segmentation process or for the searching of pattern of edges. This process of segmentation will make the clusters of the same patterns and then the edges will be detected. The initial seed points were chosen by comparing the values of intensities as the pixel with the highest intensity value will be the starting point. The membership value of each pixel is calculated and then it is assigned to specific cluster. Figure 6 shows the corresponding preprocessed image given as input both one normal and abnormal image and the corresponding result of segmentation. 
International Journal of Healthcare Information Systems and Informatics

Volume $15 \cdot$ Issue 2 • April-June 2020

Figure 6a. Normal image

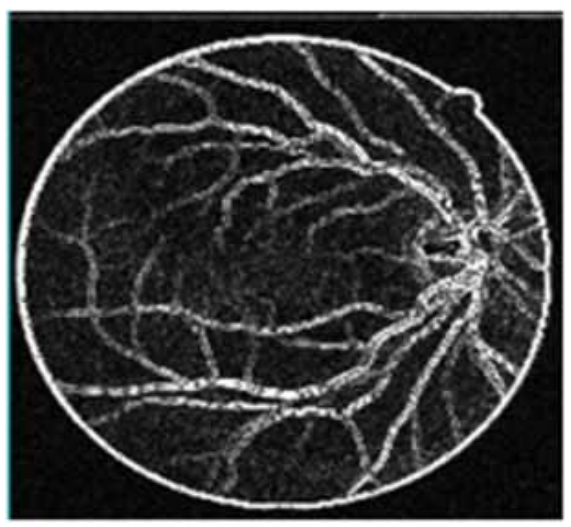

Figure 6 b. Segmented image

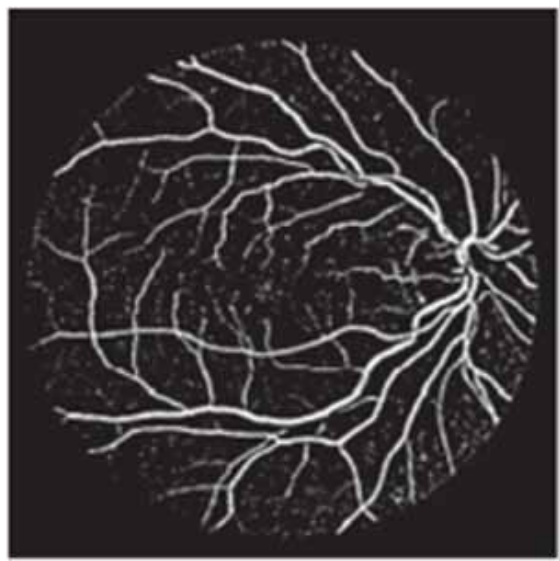

Figure 6c. Abnormal image

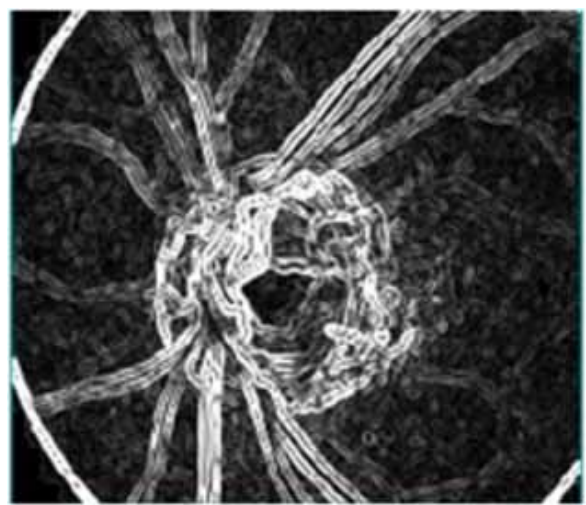




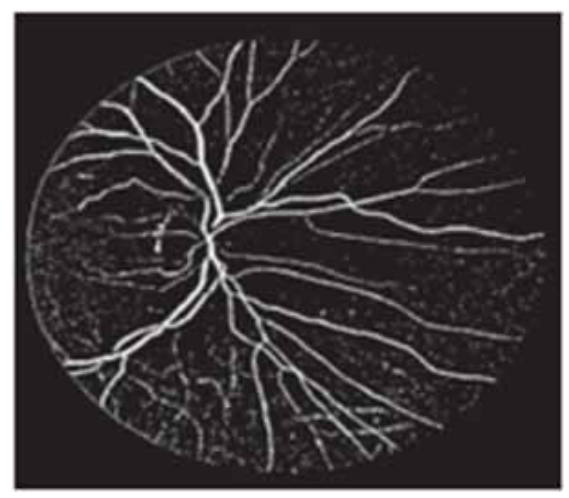

\section{Feature Extraction}

The quantitative information is detected in the process of feature extraction. The features of all the segmented images need to be extracted so that the proposed system can be trained for detection of blood vessels and for differentiating between vessels and non-vessels. Features are generally defined as attributes or properties of various segmented pixels. The feature vector for both the normal and pathological image pixels is made so that the individual results can be maintained. Also, the input image has number of features; the process of feature extraction will reduce the dimensions of the extracted features.

The process of feature extraction is done by using Independent Component Analysis (ICA) which is a statistical technique for analysis of data by computing Independent Components (ICs) of raw data. ICA gives the best results as it first removes the correlation between the data. The advantage of using ICA is that it generates statistically and non-Gaussian ICs which are not variable to location and change in phase.

Algorithm 1. Independent Component Analysis (ICA)

Input: Segmented Image

Output: Extracted features for both healthy and pathological retina. Step 1: Calculate mean and covariance matrix of the image.

step 2: The Independent Components of the raw feature vector will be extracted after performing

step 2.1: The Centering and Whitening version of the input matrix is calculated.

step 2.2: The features were extracted at random points after giving them random weights and adjusting it accordingly. The weights are adjusted by computing the values of Negentropy which is modified version of Entropy.

Step 2.3: The transformation matrix of the values is computed by decorrelating the weight matrices using decomposition method.

Step 3: The ICs computed in step 2 are saved separately for healthy and pathological retina. Figure 7 and 8 shows the feature vector plot for both healthy and pathological retina. 
Figure 7. Plot of feature vector for healthy retina

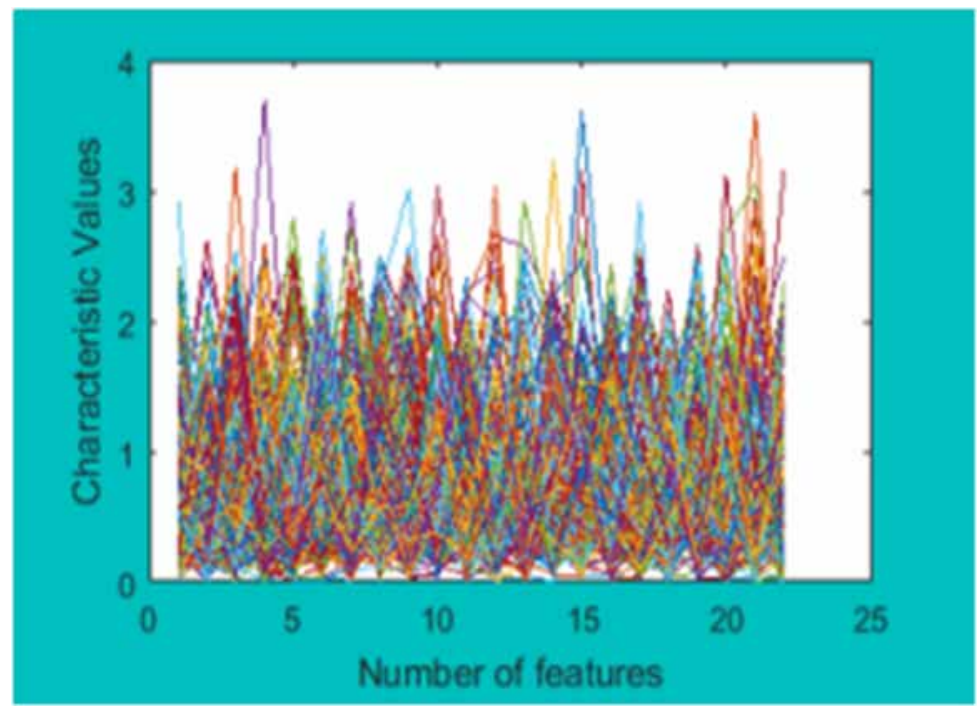

Figure 8. Plot of feature vector for pathological retina

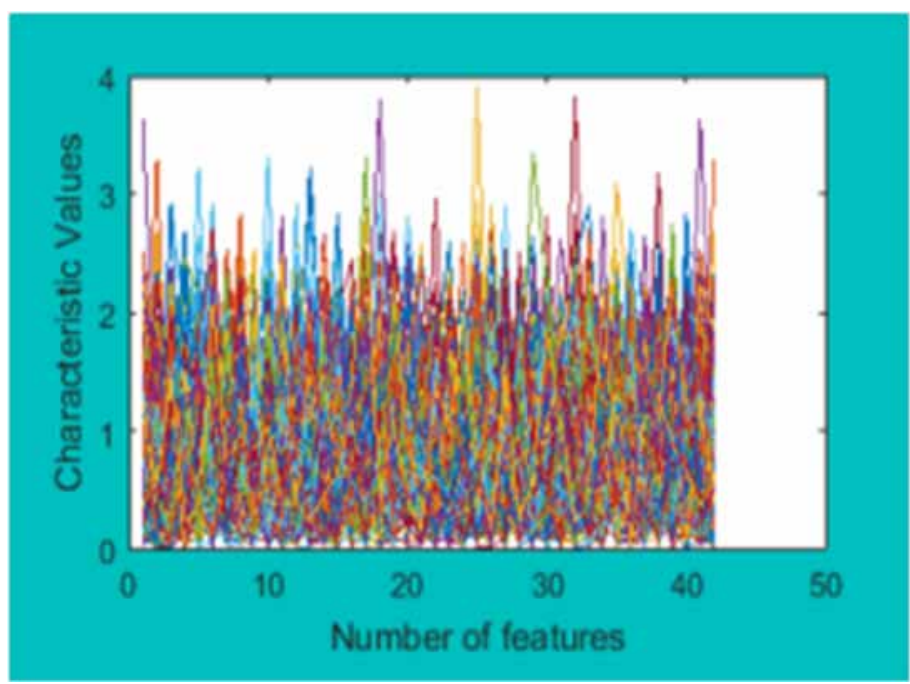

\section{Feature Optimization}

The step of feature optimization helps in optimizing the solution from all the candidate solutions. The objective function is defined for the problem and feature optimization will be performed after maximizing and minimizing the objective function. The optimization algorithms are required for dealing with image data of larger size. They only select the prominent features or optimize the data according to their value and priority. Like in this problem, the features which will be helpful in diagnosing DR will be selected and all other will not be shown in final image.

In the proposed algorithm, the features were optimized one by one using Particle Swarm Optimization (PSO), Firefly Algorithm (FA), Lion Optimization Algorithm (LOA) and Entropy based optimization algorithm. These all are bio-inspired methods of optimization. PSO predicts 
the solution of any problem using the behavior of birds flocking and swarming. The best solution is provided after computing values of velocity of the candidates. Both the local best and global best solution is provided by PSO (Singh and Pandey, 2014). Firefly algorithm is based on the behavior of fireflies which attract towards the brightness and the solutions are found by random walking of the flies and insects. LOA is another bio-inspired optimization algorithm that is based on behavior of lions. The special attraction of behavior of lion is their cooperation behavior for finding mates and preys. Figure $9 \mathrm{a}, 9 \mathrm{~b}$ and $9 \mathrm{c}$ shows the optimized plots of features after the optimization of feature vector with the help of PSO, FA and LOA.

The proposed algorithm named as Entropy based optimized segmentation (EBOS) optimize the extracted features are optimized using the values of entropy. Entropy is a measure of dispersion of histogram. Entropy will define the uncertainty of any random variable. If the image is highly ordered, then the values will be low. The entropy values for local as well as global neighborhood are calculated and final optimized values are calculated. Figure 10 shows the optimized feature vector.

\section{Feature Classification}

During the process of feature classification, the optimized features are assigned to specific target class. There will be two target classes in this case, one will be NORMAL class of images of healthy retina

Figure 9a. Optimization plot after PSO

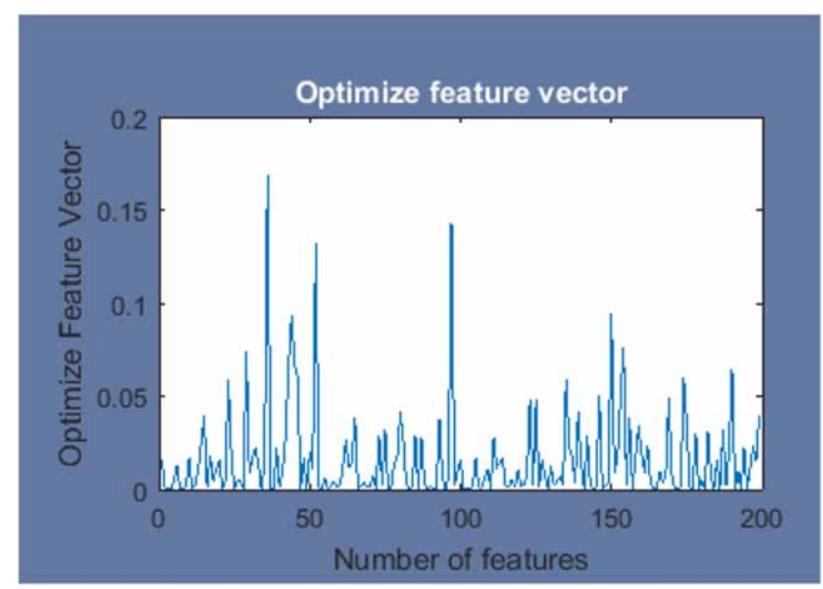

Figure 9b. Optimization plot after FA

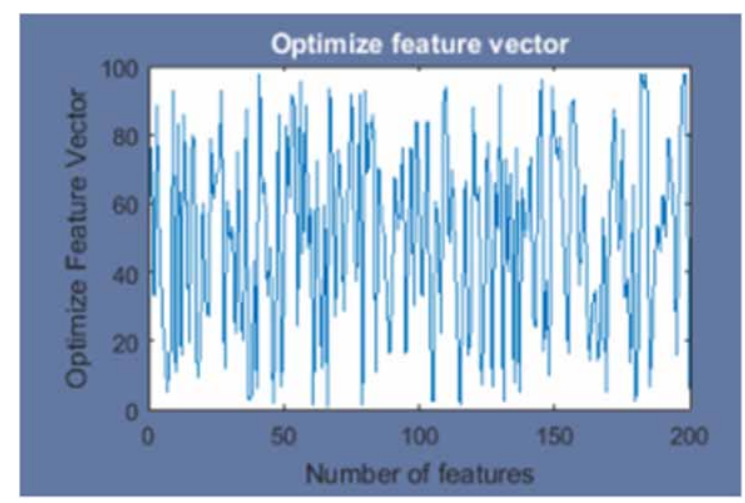


Figure 9c. Optimization plot after LOA

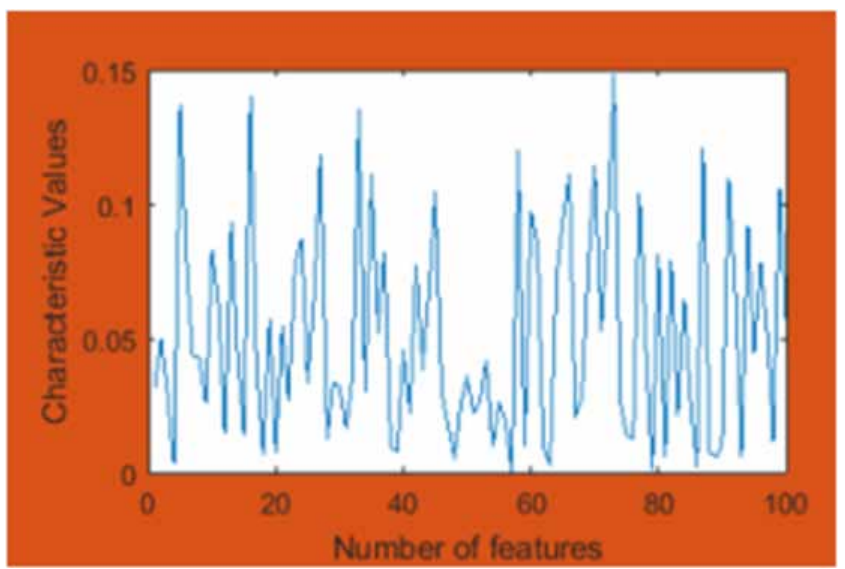

Figure 10. Optimization plot after EBOS

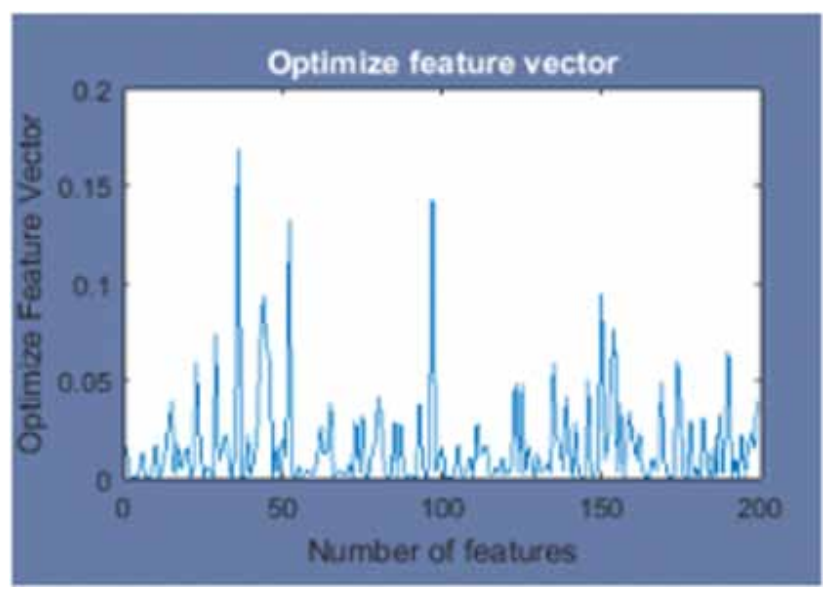

and another one will be ABNORMAL class of pathological retina. It is main phase of supervised learning as it helps in classifying the extracted features into the target class and the corresponding images as of healthy and pathological retina. The features in the proposed algorithm were classified using Support Vector Machines (SVM) (Takkar Singh, \& Pandey, 2017) which is a superior process of classification using the principle of minimization of risks. The input features are divided into different target classes using hyperplanes and the difference between hyperplane and corresponding target class.

\section{Comparison of Results}

The proposed optimization algorithm that is EBOS was compared with all the existing optimization techniques that are PSO, FA and Lion using the parameters given by Accuracy, Sensitivity, Specificity, Positive Predictive Value and False Positive Rate. The values of all the above parameters can be calculated using True Positive (TP), False Positive (FP), True Negative (TN), False Negative (FN). TP represents the pixels which are vessels in both manually segmentation as well as in segmentation by proposed algorithm. FP represents the pixels which are non-vessels in ground truth images, but vessels in proposed algorithm segmentation. TN represents the pixels which are non-vessels in both 
manually segmentation as well as in segmentation by proposed algorithm. FN represents the pixels which are vessels in ground truth images, but non-vessels in proposed algorithm segmentation.

Accuracy is defined as number of correctly classified pixels both vessels and non-vessels with respect to total number of pixels. Sensitivity is defined as ratio of correctly classified vessels with respect to total number of pixels. Specificity is defined as ratio of correctly classified non-vessels with respect to total number of pixels. Positive Predictive Value (PPV) is the ability of any algorithm for classification of vessels is really a vessel. False Positive Rate (FPR) is the ratio of pixels which are detected as vessel pixel due to some error.

All the algorithms were implemented on DRIVE, STARE and CHASE_DB1 databases. Figure 11 shows the graph of accuracy which shows the comparison of Firefly, PSO, Lion and EBOS algorithm for DRIVE database.

Figure 12 shows the graph of Sensitivity for all the four algorithms for STARE database. Figure 13 shows the graph of specificity for all for PPV and Figure 14 shows the graph of FPR value for DRIVE and Figure 15 shows the graph of FPR value.

The objective evaluation of the algorithm states that the accuracy of EBOS algorithm is higher than Firefly, PSO and Lion algorithm. Firefly is the lowest amongst all. The average accuracy achieved by the proposed algorithm is $99.37 \%$ for DRIVE, $99.13 \%$ for STARE and $99.26 \%$ for CHASE_DB1, respectively. The values of sensitivity, positive predictive value, false positive rate and specificity are also better in case of all three databases.

\section{CONCLUSION AND FUTURE SCOPE}

In this article, the segmentation of blood vessels of the human retina is performed by EBOS algorithm in which the entropy of the features is calculated individually for the optimization of the feature vector. EBOS algorithm consists of preprocessing phase, segmentation phase, feature extraction and feature optimization, feature classification and objective evaluation. The objective evaluation was performed using accuracy, sensitivity, specificity, false positive rate and positive predictive value. The values of parameters exhibit that the proposed algorithm outperformed all other algorithms. The proposed algorithm achieves the average accuracy $99.37 \%$ for DRIVE,

\section{Figure 11. Accuracy graph}

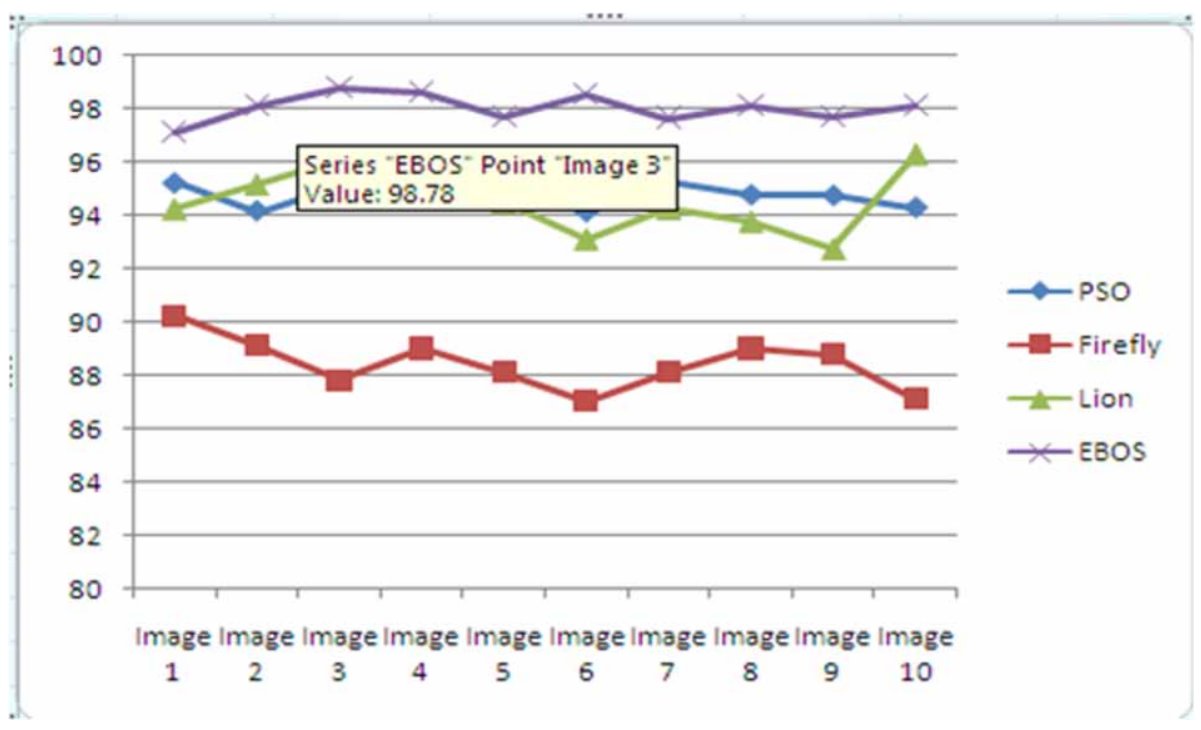


Figure 12. Sensitivity graph

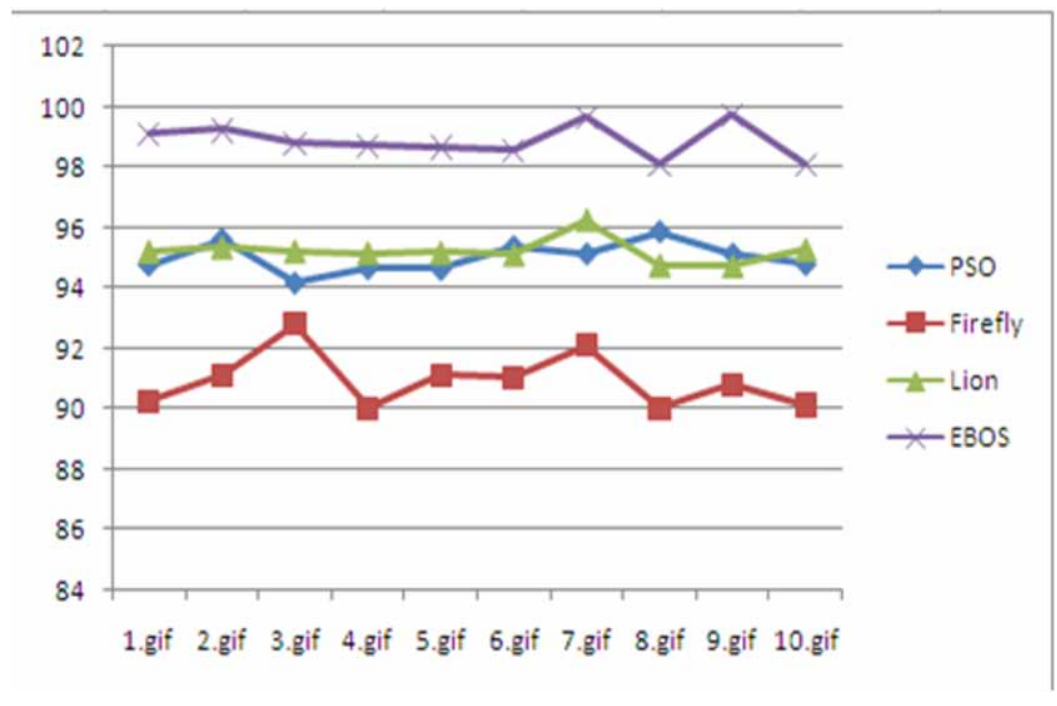

Figure 13. Graph of positive predictive value

\section{PPV - DRIVE}

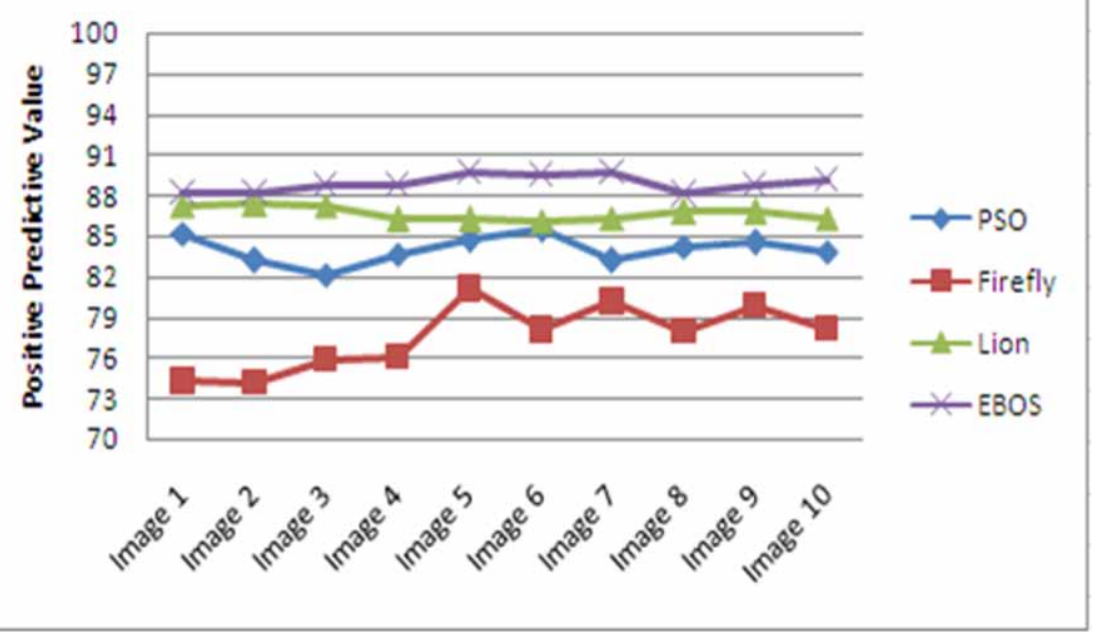

99.13\% for STARE and $99.26 \%$ for CHASE_DB1 respectively. The average sensitivity is $99.83 \%$ for DRIVE database, $99.53 \%$ for STARE and $99.12 \%$ for CHASE_DB1 respectively. EBOS algorithm was able to perform so well for these images as it is based on entropy which classifies and optimizes the images by calculating the disorder-ness in the segmented image. In future, optimization of feature vector is done for achieving $100 \%$ accuracy of the segmentation. This work can be further improved by segmenting all the symptoms of DR in the retina and predicting 


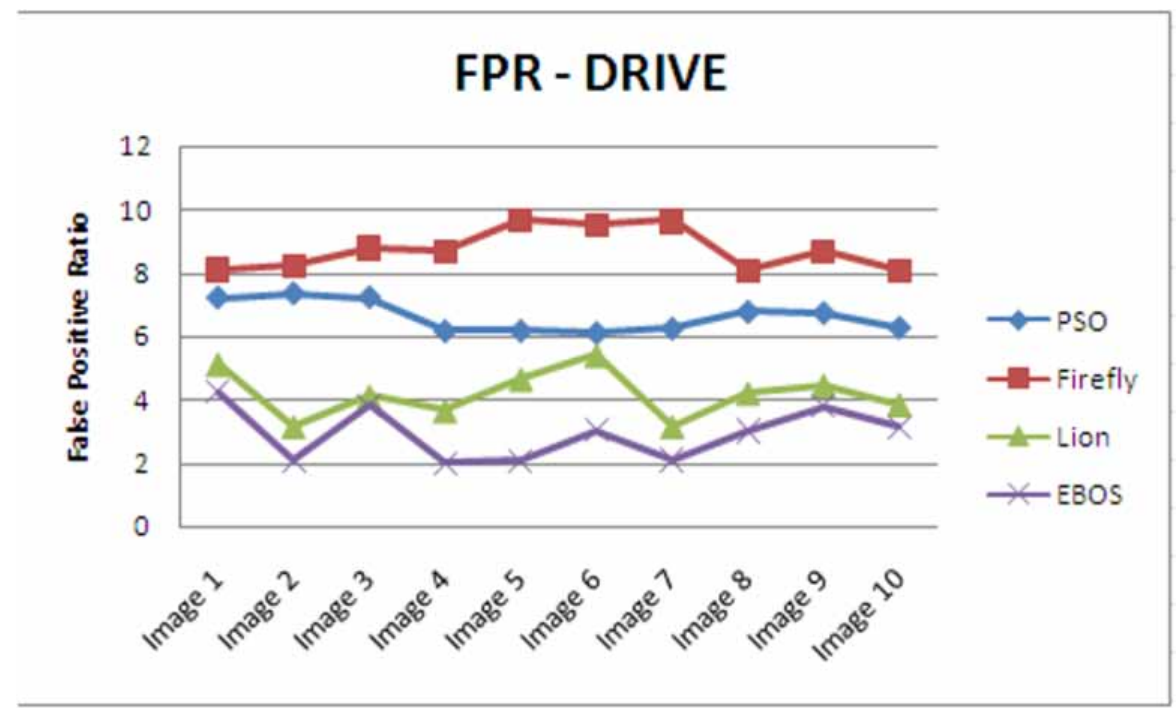

Figure 15. Graph of specificity

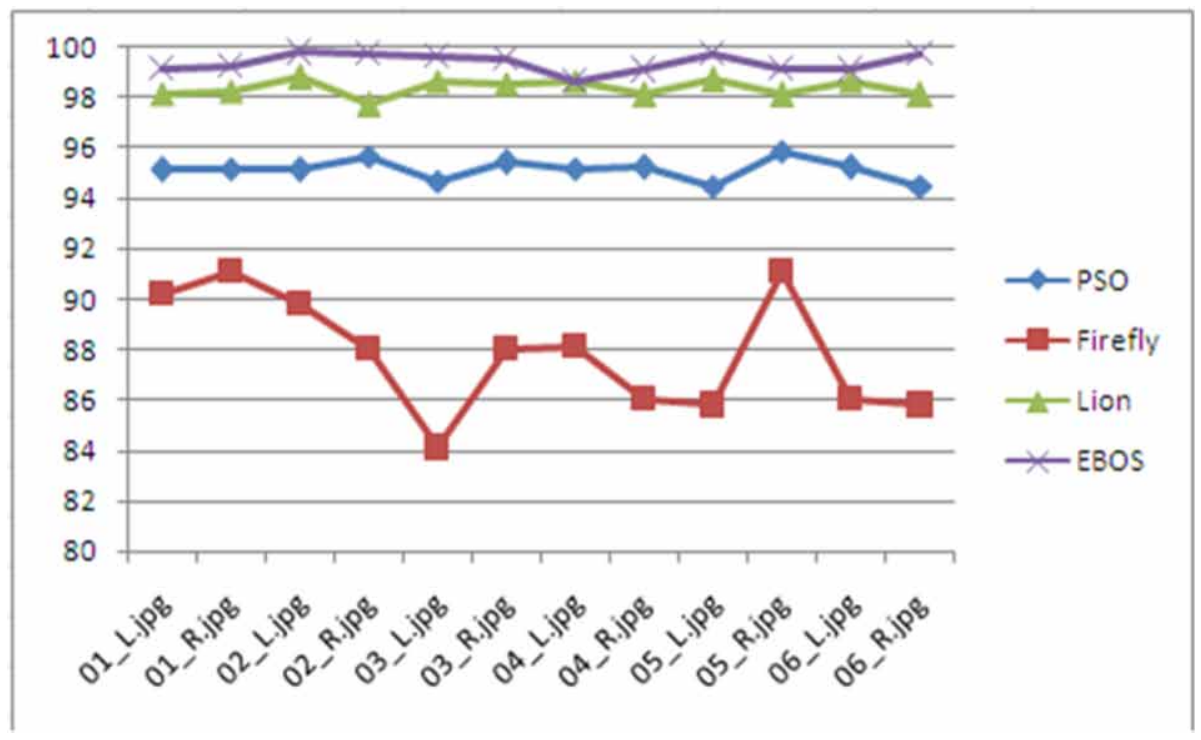

the specific stage of DR. Different optimization techniques can be further applied on segmented images for achieving accuracy.

\section{ACKNOWLEDGMENT}

The authors are thankful to IKG Punjab Technical University, Kapurthala, Punjab to give the opportunity to do this research work. 


\section{REFERENCES}

Anzalone, A., Bizzarri, F., Parodi, M., \& Storace, M. (2008). A modular supervised algorithm for vessel segmentation in red-free retinal images. Computers in Biology and Medicine, 38(8), 913-922.

Aslani, S., \& Sarnel, H. (2016). A new supervised retinal vessel segmentation method based on robust hybrid features. Biomedical Signal Processing and Control, 30, 1-12.

Franklin, S. W., \& Rajan, S. E. (2014). Computerized screening of diabetic retinopathy employing blood vessel segmentation in retinal images. Biocybernetics and Biomedical Engineering, 34(2), 117-124.

Franklin, S. W., \& Rajan, S. E. (2014). Retinal vessel segmentation employing ANN technique by Gabor and moment invariants-based features. Applied Soft Computing, 22, 94-100.

Fraz, M. M., Remagnino, P., Hoppe, A., Uyyanonvara, B., Rudnicka, A. R., Owen, C. G., \& Barman, S. A. (2012). Blood vessel segmentation methodologies in retinal images-a survey. Computer Methods and Programs in Biomedicine, 108(1), 407-433.

GeethaRamani, R., \& Balasubramanian, L. (2016). Retinal blood vessel segmentation employing image processing and data mining techniques for computerized retinal image analysis. Biocybernetics and Biomedical Engineering, 36(1), 102-118.

Hatanaka, Y., Samo, K., Tajima, M., Ogohara, K., Muramatsu, C., Okumura, S., \& Fujita, H. (2016, March). Automated blood vessel extraction using local features on retinal images. In Medical Imaging 2016: ComputerAided Diagnosis (p. 97852F). International Society for Optics and Photonics.

Henkind, P., \& Wise, G. N. (1974). Retinal neovascularization, collaterals, and vascular shunts. The British Journal of Ophthalmology, 58(4), 413-422.

Hoyer, P. O., \& Hyvärinen, A. (2000). Independent component analysis applied to feature extraction from colour and stereo images. Network (Bristol, England), 11(3), 191-210.

Joshi, S. R. (2015). Diabetes Care in India. Annals of Global Health, 81(6), 830-838.

Li, M., Zhenshen, M., Chao, L., Guang, Z., \& Zhe, H. (2017). Robust retinal blood vessel segmentation based on reinforcement local descriptions. BioMed Research International. doi:10.1155/2017/2028946

Lupascu, C. A., Tegolo, D., \& Trucco, E. (2010). FABC: Retinal vessel segmentation using AdaBoost. IEEE Transactions on Information Technology in Biomedicine, 14(5), 1267-1274.

Marín, D., Aquino, A., Gegúndez-Arias, M. E., \& Bravo, J. M. (2010). A new supervised method for blood vessel segmentation in retinal images by using gray-level and moment invariants-based features. IEEE Transactions on Medical Imaging, 30(1), 146-158.

Marini, F., \& Walczak, B. (2015). Particle swarm optimization (PSO). A tutorial. Chemometrics and Intelligent Laboratory Systems, 149, 153-165.

Mustafa, W. A., Haniza, Y., \& Wahida, K. (2017). Combination of gray-level and moment invariant for automatic blood vessel detection on retinal image. Journal of Biometrics. Biomaterials and Biomedical Engineering, 34, $10-19$.

Osareh, A., \& Shadgar, B. (2009). Automatic blood vessel segmentation in color images of retina. Indian Journal of Science and Technology, 33(B2), 191.

Rahim, S. S., Jayne, C., Palade, V., \& Shuttleworth, J. (2016). Automatic detection of microaneurysms in colour fundus images for diabetic retinopathy screening. Neural Computing \& Applications, 27(5), 1149-1164.

Rajput, M. Y., Manza, R. R., Patwari, M. B., Rathod, D. D., Borde, P. L., \& Yannawal, P. L. (2015). Detection of Non-Proliferative Diabetic Retinopathy lesions using wavelet and classification using k-means clustering. In Proceedings of the International Conference on Communication Networks (pp. 381-387). IEEE. doi:10.1109/ ICCN.2015.73

Ricci, E., \& Perfetti, R. (2007). Retinal blood vessel segmentation using line operators and support vector classification. IEEE Transactions on Medical Imaging, 26(10), 1357-1365. 
Roychowdhury, S., Koozekanani, D. D., \& Parhi, K. K. (2014). Blood vessel segmentation of fundus images by major vessel extraction and subimage classification. IEEE Journal of Biomedical and Health Informatics, 19(3), 1118-1128. doi:10.1109/JBHI.2014.2335617

Saha, M., Naskar, M. K., \& Chatterji, B. N. (2016). Detection of Diabetic Retinopathy Using the Wavelet Transform and Feedforward Neural Network. In Advanced Computing and Systems for Security (pp. 101-109). New Delhi: Springer; . doi:10.1007/978-81-322-2650-5_7

Shah, S. A. A., Tang, T. B., Faye, I., \& Laude, A. (2017). Blood vessel segmentation in color fundus images based on regional and Hessian features. Graefes Archive for Clinical and Experimental Ophthalmology, 255(8), 1525-1533.

Singh, A., \& Pandey, B. (2014). Intelligent techniques and applications in liver disorders: A survey. International Journal of Biomedical Engineering and Technology, 16(1), 27-70. doi:10.1504/IJBET.2014.065638

Sinthanayothin, C., Boyce, J. F., Cook, H. L., \& Williamson, T. H. (1999). Automated localisation of the optic disc, fovea, and retinal blood vessels from digital colour fundus images. The British Journal of Ophthalmology, 83(8), 902-910.

Soares, J. V., Leandro, J. J., Cesar, R. M., Jelinek, H. F., \& Cree, M. J. (2006). Retinal vessel segmentation using the 2-D Gabor wavelet and supervised classification. IEEE Transactions on Medical Imaging, 25(9), 1214-1222. doi:10.1109/TMI.2006.879967

Staal, J., Abràmoff, M. D., Niemeijer, M., Viergever, M. A., \& Van Ginneken, B. (2004). Ridge-based vessel segmentation in color images of the retina. IEEE Transactions on Medical Imaging, 23(4), 501-509. doi:10.1109/ TMI.2004.825627

Takkar, S., Singh, A., \& Pandey, B. (2017). Application of machine learning algorithms to a well defined clinical problem: Liver disease. International Journal of E-Health and Medical Communications, 8(4), 38-60. doi:10.4018/IJEHMC.2017100103

Tang, S., Lin, T., Yang, J., Fan, J., Ai, D., \& Wang, Y. (2015). Retinal vessel segmentation using supervised classification based on multi-scale vessel filtering and Gabor wavelet. Journal of Medical Imaging and Health Informatics, 5(7), 1571-1574.

Wang, S., Yin, Y., Cao, G., Wei, B., Zheng, Y., \& Yang, G. (2015). Hierarchical retinal blood vessel segmentation based on feature and ensemble learning. Neurocomputing, 149, 708-717.

Xu, L., \& Luo, S. (2010). A novel method for blood vessel detection from retinal images. Biomedical Engineering Online, 9(1), 14. doi:10.1186/1475-925X-9-14

You, X., Peng, Q., Yuan, Y., Cheung, Y. M., \& Lei, J. (2011). Segmentation of retinal blood vessels using the radial projection and semi-supervised approach. Pattern Recognition, 44(10-11), 2314-2324.

Zimmet, P. Z., \& Alberti, K. G. M. (2016). Epidemiology of diabetes—status of a pandemic and issues around metabolic surgery. Diabetes Care, 39(6), 878-883.

Sukhpreet Kaur is pursuing a Ph.D from IKG Punjab Technical University in Digital Image Processing. She did her $B$. Tech and M.tech in Computer Science and Engineering.

Kulwinder Singh Mann completed a Ph.D from IKG Punjab Technical University in Medical Informatics. He did his B. Tech and M.tech in Computer Science and Engineering. Currently, he is a Professor in the IT department of Guru Nanak Dev Engineering College, Ludhiana. 